\section{Computer analysiert Kratzverhalten}

Juckreiz ist ein Hauptsymptom des atopischen Ekzems sowie ein wichtiger Marker für die Krankheitsaktivität. Seine Intensität wurde bisher überwiegend durch Fragebögen oder visuelle Analogskalen ermittelt. Eine Pilotstudie versuchte nun, das nächtliche Kratzverhalten von Kindern mit Neurodermitits zu objektivieren.

B ritische Dermatologen untersuchten 14 Kinder im Alter zwischen 2 und 9 Jahren mit einem schweren atopischen Ekzem sowie sieben gesunde Kontrollkinder im Alter zwischen 5 und 7 Jahren. An ihren Armen und Beinen wurden über Nacht rund $20 \mathrm{~g}$ schwere digitale Bewegungsmesser angebracht, deren Signale auf einen Computer übertragen wurden. Als Goldstandard für die Bewegungsmessung dienten InfrarotVideoaufnahmen während des Schlafes.

Das Schlafverhalten der beiden Gruppen unterschied sich deutlich: Die von Juckreiz geplagten Kinder verbrachten pro Nacht im Durchschnitt $46 \mathrm{Mi}$ nuten weniger schlafend oder ruhig liegend als die Kontrollkinder. Außerdem zeigten die kleinen Ekzempatienten zwei- bis dreimal häufiger Kratzepisoden oder ein ruheloses Umherwerfen im Bett. Die Unterschiede waren jeweils signifikant. Kratzen und Ruhelosigkeit waren eng miteinander korreliert. Die mit den Bewegungsmessern erhobenen Befunde stimmten auch gut mit den $\mathrm{Vi}$ deoaufnahmen überein. Die Bewegungen der Arme bzw. der Beine erfolgten relativ synchron, so dass die Messung an jeweils einer Extremität zur Dokumentation ausgereicht hätte. Die ermittelten Daten korrelierten allerdings wenig mit den Angaben der Mütter, die auf einer visuellen Analogskala über das vermutete Kratzverhalten ihrer Kinder während der Nacht Auskunft geben sollten.

Fazit: An den Extremitäten angebrachte Bewegungsmesser dokumentieren zuverlässig das Kratz- und Bewegungs-

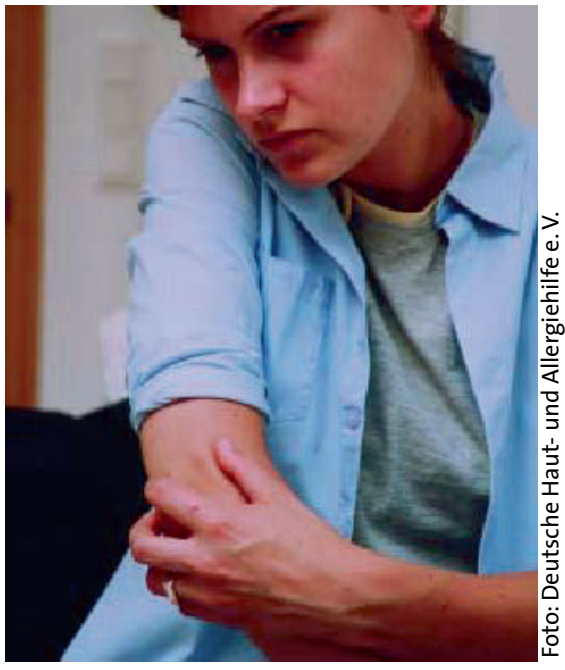

Kann man „Kratzen“ messen? Britische Dermatologen haben es versucht.

muster während des Schlafs und sind somit ein indirektes Messinstrument für nächtlichen Juckreiz bei Kindern mit atopischer Dermatitis.

$b k$

Benjamin K et al. The development of an objective method for measuring scratch in children with atopic dermatitis suitable for clinical use. J Am Acad Dermatol 2004; 50: 33-40

\title{
Calcineurininhibitor auch für die Kleinsten
}

\section{Der topische Immunmodulator Pimecrolimus hat sich als wirksam und verträglich in der Therapie von Patienten mit atopischem Ekzem erwiesen. Eine gepoolte Analyse überprüfte jetzt die Unbedenk- lichkeit der Substanz speziell bei einem Einsatz im Kleinkindesalter.}

E nglische Ärzte untersuchten in drei offenen Studien Kinder zwischen 8 und 14 Jahren $(n=10$, Studie A), Kleinkinder zwischen 8 und 30 Monaten $(\mathrm{n}=$ 8, Studie B) und Säuglinge zwischen 4 und 11 Monaten $(\mathrm{n}=8$, Studie C) mit atopischem Ekzem. Alle Probanden erhielten 1\%ige Pimecrolimus-Creme zweimal täglich über 22 Tage aufgetragen, die Serumkonzentrationen von Pimecrolimus wurden bestimmt und die Wirksamkeit anhand des Eczema Area and Severity Index (EASI) beurteilt.

Insgesamt waren in den Studien A und $\mathrm{B}$ die Serumkonzentrationen von
Pimecrolimus niedrig: Sie lagen bei $81 \%$ der Messungen unter $1 \mathrm{ng} / \mathrm{ml}$, bei der Hälfte der Proben wurde die Nachweisgrenze von $0,5 \mathrm{ng} / \mathrm{ml}$ nicht erreicht. Den höchsten Wert mit 2,5 ng/ml wurde bei einem Kind aus der Studie C 2 Stunden nach dem Auftragen an Tag 4 gemessen. Der Tag 4 war bei den meisten Kindern der Tag der höchsten systemischen Pimecrolimus-Konzentration, da spätestens ab diesem Zeitpunkt die Abheilung einsetzte und damit die Barrierefunktion der Haut sich wieder verbesserte. Die lokale Verträglichkeit der Creme war gut. Bei fünf der 26 kleinen
Patienten trat ein leichtes bis mittelschweres Brennen an der Applikationsstelle auf. Eine systemische Akkumulation des Wirkstoffs fand nicht statt.

Der Therapieeffekt machte sich innerhalb von 4 Tagen bemerkbar, der mediane Score-Rückgang im EASI am Tag 22 gegenüber Therapiebeginn betrug 55\% (Studie A), 63\% (Studie B) bzw. 83\% (Studie C). Der Therapieeffekt im Vergleich zu den Ausgangsbefunden war zu allen Kontrollzeitpunkten statistisch signifikant $(\mathrm{p}<0,0001)$.

Fazit: Eine 3-wöchige Behandlung mit Pimecrolimus wird auch von sehr jungen Kindern mit atopischem Ekzem gut vertragen, die systemische Belastung durch die Substanz ist gering. $\quad b k$

Allen BR et al. Systemic exposure, tolerability, and efficacy of pimecrolimus cream $1 \%$ in atopic dermatitis patients. Arch Dis Child 2003; 113: 969-73 